\title{
High Fibrosis-4 Index Is Related with Worse Clinical Outcome in Patients with Coronavirus Disease 2019 and Diabetes Mellitus: A Multicenter Observational Study
}

\author{
Sung-Woo Kim ${ }^{1, *}$, Jae-Han Jeon²,*, Jun Sung Moon ${ }^{3}$, Mi Kyung Kim ${ }^{4}$ \\ ${ }^{1}$ Department of Internal Medicine, Daegu Catholic University Hospital, Daegu Catholic University School of Medicine; \\ ${ }^{2}$ Department of Internal Medicine, Kyungpook National University Chilgok Hospital, School of Medicine, Kyungpook National \\ University; ${ }^{3}$ Department of Internal Medicine, Yeungnam University Hospital, Yeungnam University College of Medicine; \\ ${ }^{4}$ Department of Internal Medicine, Keimyung University Dongsan Hospital, Keimyung University School of Medicine, Daegu, \\ Korea
}

Background: Based on recent evidence on the importance of the presence of diabetes mellitus (DM) and fibrosis-4 (FIB-4) index in coronavirus disease 2019 (COVID-19) mortality, we analyzed whether these factors could additively predict such mortality.

Methods: This multicenter observational study included 1,019 adult inpatients admitted to university hospitals in Daegu. The demographic and laboratory findings, mortality, prevalence of severe disease, and duration of quarantine were compared between patients with and without DM and/or a high FIB-4 index. The mortality risk and corresponding hazard ratio (HR) were analyzed using the Kaplan-Meier method and Cox proportional hazard models.

Results: The patients with DM $(n=217)$ exhibited significantly higher FIB-4 index and mortality compared to those without DM. Although DM (HR, 2.66; 95\% confidence interval [CI], 1.63 to 4.33) and a high FIB-4 index (HR, 4.20; 95\% CI, 2.21 to 7.99) were separately identified as risk factors for COVID-19 mortality, the patients with both DM and high FIB-4 index had a significantly higher mortality (HR, 9.54; 95\% CI, 4.11 to 22.15). Higher FIB-4 indices were associated with higher mortality regardless of DM. A high FIB-4 index with DM was more significantly associated with a severe clinical course with mortality (odds ratio, $11.24 ; 95 \%$ CI, 5.90 to 21.41) than a low FIB-4 index without DM, followed by a high FIB-4 index alone and DM alone. The duration of quarantine and hospital stay also tended to be longer in those with both DM and high FIB-4 index.

Conclusion: Both DM and high FIB-4 index are independent and additive risk factors for COVID-19 mortality.

Keywords: Non-alcoholic fatty liver disease; Diabetes mellitus; COVID-19; Mortality

Received: 17 March 2021, Revised: 25 May 2021, Accepted: 31 May 2021

Corresponding authors: Mi Kyung Kim

Division of Endocrinology and Metabolism, Department of Internal Medicine, Keimyung University Dongsan Hospital, Keimyung University School of Medicine, 1035 Dalgubeol-daero, Dalseo-gu, Daegu 42601, Korea Tel: +82-53-258-7730, Fax: +82-53-258-4990, E-mail: mdkmk@dsmc.or.kr

Jun Sung Moon

Division of Endocrinology and Metabolism, Department of Internal Medicine, Yeungnam University Hospital, Yeungnam University College of Medicine, 170 Hyeonchung-ro, Nam-gu, Daegu 42415, Korea

Tel: +82-53-620-3825, Fax: +82-53-654-3486, E-mail: mjs7912@yu.ac.kr
Copyright $\odot 2021$ Korean Endocrine Society

This is an Open Access article distributed under the terms of the Creative Commons Attribution Non-Commercial License (https://creativecommons.org/ licenses/by-nc/4.0/) which permits unrestricted non-commercial use, distribution, and reproduction in any medium, provided the original work is properly cited.

*These authors contributed equally to this work. 


\section{INTRODUCTION}

Since the first report of coronavirus disease 2019 (COVID-19) caused by severe acute respiratory syndrome coronavirus 2 (SARS-CoV-2), it has spread worldwide, with more than 100 million individuals infected. COVID-19 has a broad spectrum of clinical manifestations, ranging from no symptoms at all to critical illness. Most COVID-19 cases are mild; however, according to reports, approximately $20 \%$ of cases progress to severe cases [1]. Old age and presence of comorbidities, such as diabetes mellitus (DM) or cardiovascular disease, have been reported as risk factors for progression to severe cases and mortality $[2,3]$. In addition, a recent report showed that patients with severe disease have abnormal liver function test results as well as inflammation markers, and abnormal liver function test results are reported as risk factors for progression to severe disease $[4,5]$.

Non-alcoholic fatty liver disease (NAFLD) is one of the most common liver diseases worldwide and is a leading cause of advanced liver disease, cirrhosis, and hepatocellular carcinoma [6]. It is associated with overweightness/obesity or the presence of type $2 \mathrm{DM}$ or evidence of metabolic dysregulation [7]. Therefore, some experts have proposed a change in nomenclature into metabolic-associated fatty liver disease (MAFLD) $[7,8]$. Recent studies have shown that metabolic abnormalities related to NAFLD, such as obesity and DM, are associated with severe COVID-19 [2,3,6,9] and MAFLD has also been reported as a risk factor for severe COVID-19 [10,11]. Moreover, among patients with MAFLD with a high fibrosis-4 (FIB-4) index, noninvasive fibrosis markers are associated with a high risk for severe COVID-19 irrespective of metabolic comorbidities [12]. Our previous observation was consistent with other findings; high aspartate aminotransferase (AST) levels were found to be related to both severe clinical course and mortality, irrespective of the history of DM [9]. In addition, FIB-4 was independently associated with risks of all-cause mortality in atrial fibrillation patients $[13,14]$, and even in the general population [15]. Recently, there have been reports that FIB-4 may be a predictor of prognosis in COVID-19 patients [16] regardless of the presence of NAFLD/non-alcoholic steatohepatitis (NASH), but the results were not conclusive $[17,18]$. This prompted us to determine whether the FIB-4 index affects the severity of or mortality from COVID-19 in patients with and without DM.

\section{METHODS}

\section{Study design and participants}

This study was a multicenter, retrospective, observational study. The study protocol was approved by the Institutional Review Boards of Keimyung University Dongsan Hospital (2020-04111 ) and each participating hospital. The need for informed consent was waived by the ethics boards of the hospitals because of the retrospective study design.

The study participants comprised adult patients (age $>18$ years) with laboratory-confirmed COVID-19 between February 18, 2020 and March 31, 2020. They were recruited from tertiary hospitals (Daegu Catholic University Hospital, Keimyung University Dongsan Medical Center [Dongsan Hospital and Daegu Dongsan Hospital], Kyungpook National University Hospital, Kyungpook National University Chilgok Hospital, and Yeungnam University Hospital) in Daegu.

COVID-19 was diagnosed using a reverse transcription-polymerase chain reaction (RT-PCR) assay of nasal and pharyngeal swab specimens, and a confirmed case was defined by a positive RT-PCR result, irrespective of the clinical signs and symptoms present. A total of 1,100 patients were initially included in this study. Patients who were diagnosed with COVID-19 re-infection were excluded from the study. Patients who were admitted to more than two hospitals because of referral within the cohort were counted as a single case. To exclude the effect of acute liver injury, we also excluded patients who had an ALT level of $\geq 80 \mathrm{IU} / \mathrm{L}$ at the time of admission or died within 3 days from diagnosis with COVID-19. After exclusion, 1,019 patients were finally enrolled in the study (Table 1).

\section{Data collection and definitions}

We collected data from the electronic medical records of the patients, including age, sex, vital signs, comorbidities (hypertension, cerebrovascular disease, cardiovascular disease, chronic kidney disease $[\mathrm{CKD}]$, chronic pulmonary disease, dialysis requirement, and cancer), medication, laboratory findings, presence of pneumonia on chest radiography or computed tomography, treatment modality, and hospitalization outcomes. The major symptoms present (fever, cough, sputum, rhinorrhea, sore throat, myalgia, diarrhea, and shortness of breath) were also recorded. The laboratory findings included routine blood test results, glycated hemoglobin A1c level, blood glucose level, C-reactive protein level, procalcitonin level, and serum indicators of liver or kidney injury. The presence of DM was defined based on the participants' medical history and the diagnostic criteria 
Table 1. Baseline Characteristics in Patients with COVID-19

\begin{tabular}{lccc}
\hline Characteristic & $\begin{array}{c}\text { Non-DM } \\
(n=802)\end{array}$ & $\begin{array}{c}\text { DM } \\
(n=217)\end{array}$ & $P$ value \\
\hline Age, yr & $56.4 \pm 18.0$ & $68.7 \pm 11.2$ & $<0.01$ \\
Male sex & $257(32.0)$ & $95(43.8)$ & $<0.01$ \\
FIB-4 index & $1.95 \pm 2.19$ & $2.93 \pm 3.76$ & $<0.01$ \\
High FIB-4 index & $181(22.6)$ & $88(40.6)$ & $<0.01$ \\
Co-morbidity & & & \\
Hypertension & $220(27.4)$ & $136(62.7)$ & $<0.01$ \\
Heart disease & $47(5.9)$ & $27(11.1)$ & $<0.01$ \\
Cerebro-vascular disease & $40(5.0)$ & $22(10.2)$ & $<0.01$ \\
Chronic kidney disease & $14(1.7)$ & $18(8.3)$ & $<0.01$ \\
Chronic lung disease & $54(6.7)$ & $16(7.4)$ & 0.41 \\
Cancer & $42(5.2)$ & $17(7.8)$ & 0.10
\end{tabular}

Laboratory findings on admission

\begin{tabular}{|c|c|c|c|}
\hline $\mathrm{HbA} 1 \mathrm{c}, \%$ & $5.74 \pm 0.62$ & $7.63 \pm 1.75$ & $<0.01$ \\
\hline Glucose, mg/dL & $109.1 \pm 29.7$ & $184.7 \pm 103.1$ & $<0.01$ \\
\hline $\mathrm{WBC}, 10^{3} / \mu \mathrm{L}$ & $5.52 \pm 2.35$ & $6.90 \pm 3.68$ & $<0.01$ \\
\hline $\mathrm{Hb}, \mathrm{g} / \mathrm{dL}$ & $12.7 \pm 1.60$ & $12.2 \pm 1.71$ & $<0.01$ \\
\hline $\mathrm{PLT}, 10^{3} / \mu \mathrm{L}$ & $226.2 \pm 83.1$ & $232.5 \pm 101.5$ & 0.35 \\
\hline $\mathrm{CRP}, \mathrm{mg} / \mathrm{dL}$ & $6.7 \pm 20.5$ & $10.9 \pm 19.5$ & $<0.01$ \\
\hline Total protein, $\mathrm{g} / \mathrm{dL}$ & $6.77 \pm 0.55$ & $6.69 \pm 0.70$ & 0.12 \\
\hline Albumin, g/dL & $3.95 \pm 0.48$ & $3.65 \pm 0.56$ & $<0.01$ \\
\hline eGFR, $\mathrm{mL} / \mathrm{min} / 1.73 \mathrm{~m}^{2}$ & $98.4 \pm 33.9$ & $79.0 \pm 33.7$ & $<0.01$ \\
\hline AST, IU/L & $27.9 \pm 15.7$ & $33.0 \pm 22.4$ & $<0.01$ \\
\hline ALT, IU/L & $23.0 \pm 13.7$ & $23.3 \pm 13.7$ & 0.79 \\
\hline LDH, IU/L & $443.8 \pm 177.4$ & $530.7 \pm 415.7$ & $<0.01$ \\
\hline \multicolumn{4}{|l|}{ Treatment } \\
\hline ICU & $62(7.7)$ & $50(23.0)$ & $<0.01$ \\
\hline High flow $\mathrm{O}_{2}$ & $45(5.6)$ & $30(13.8)$ & $<0.01$ \\
\hline Ventilator & $31(3.9)$ & $31(14.3)$ & $<0.01$ \\
\hline CRRT & $5(0.6)$ & $13(6.0)$ & $<0.01$ \\
\hline ECMO & $4(0.5)$ & $8(3.7)$ & $<0.01$ \\
\hline \multicolumn{4}{|l|}{ Duration } \\
\hline Confirmation-release $^{\mathrm{a}}$, day & $29.3 \pm 13.5$ & $32.5 \pm 14.0$ & $<0.01$ \\
\hline Hospital length ${ }^{\mathrm{a}}$, day & $24.5 \pm 13.3$ & $28.7 \pm 14.6$ & $<0.01$ \\
\hline \multicolumn{4}{|l|}{ Outcome } \\
\hline Release & $726(90.5)$ & $170(78.3)$ & $<0.01$ \\
\hline Severe clinical course & $80(10.0)$ & $57(26.3)$ & $<0.01$ \\
\hline Death & $32(4.0)$ & $39(18.0)$ & $<0.01$ \\
\hline
\end{tabular}

Values are expressed as mean \pm standard deviation or number $(\%)$. All $P$ values were calculated using the Student's $t$ test or the chi-square test. COVID-19, coronavirus disease 2019; DM, diabetes mellitus; FIB-4, fibrosis-4; HbA1c, hemoglobin A1c; WBC, white blood cell; Hb, hemoglobin; PLT, platelet; CRP, C-reactive protein; eGFR, estimated glomerular filtration rate; AST, aspartate aminotransferase; ALT, alanine aminotransferase; $\mathrm{LDH}$, lactate dehydrogenase; ICU, intensive care unit; CRRT, continuous renal replacement therapy; ECMO, extracorporeal membrane oxygenation. ${ }^{a}$ Only includes patients who had been released from quarantine. for type $2 \mathrm{DM}$ of the Korean Diabetes Association [19]. Release from quarantine was defined using the instructions from the Korean Central Disease Control Headquarters: (1) absence of a fever, without the need for an anti-pyretic agent, and an improvement in symptoms; (2) negative results of RT-PCR tests performed twice at a 24-hour interval (http://ncov.mohw.go.kr/ baroView); and (3) severe disease defined as the necessity for the use of a high-flow nasal cannula, mechanical ventilation, continuous renal replacement therapy, or extracorporeal membrane oxygenation or admission to an intensive care unit.

The FIB-4 index was calculated using the following equation $[$ age $\times$ AST level $(\mathrm{IU} / \mathrm{L})] /\left[\left(\right.\right.$ platelet count $\left.\times 10^{9}\right) \times \sqrt{ }$ ALT level (IU/L)] based on the blood test results obtained at the time of hospital admission, before starting any specific COVID-19 therapies. Specifically, a FIB-4 index of over 3.25 or 2.0 and an age of $>65$ years were associated with a high risk for advanced fibrosis; the patients meeting these criteria were categorized into the high FIB-4 index group. Conversely, those with a FIB-4 index of below 3.25 or 2.0 aged $>65$ years were assigned to the low FIB-4 index group [20].

\section{Quantification and statistical analysis}

Statistical analyses were performed using SPSS Statistics version 18.0 (SPSS Inc., Chicago, IL, USA). Continuous data were presented as mean \pm standard deviation and categorical data as frequency rates and percentages. Comparisons between the two groups were performed using Student's $t$ test for continuous data and the chi-square test for categorical data. The risk of mortality and the corresponding hazard ratio (HR) were analyzed using the Kaplan-Meier method and Cox proportional hazard models. A multivariate logistic analysis of the initial laboratory findings was performed to identify the prognostic factors for severe disease and mortality from COVID-19. Differences were considered to be statistically significant at a two-sided $\alpha$ of $<0.05$.

\section{RESULTS}

\section{Baseline characteristics, including the FIB-4 index, according to the presence of DM}

The baseline characteristics of the patients are presented in Table 1. Based on recent literature indicating higher mortality in patients with DM [21-23] and the FIB-4 index as a novel indicator of COVID-19 mortality [16,24], we measured the FIB-4 index in the patients with COVID-19. As expected, the patients with DM had a significantly higher FIB-4 index (2.93 \pm 3.76 vs. 
$1.95 \pm 2.19, P<0.01$ ) as well as a higher mortality (Table 1). Accordingly, the proportion of patients with a high FIB-4 index was significantly higher among those with DM (40.6\% vs. $22.6 \%, P<0.01$ ) (Table 1$)$.

\section{High FIB-4 index and presence of DM are predictors of severe clinical course and mortality in patients with COVID-19}

Next, we determined the risk factors for mortality among the patients with COVID-19. The multivariate logistic analysis showed that the presence of hypertension and cancer was a risk factor for a severe clinical course and chronic lung disease for mortality. Meanwhile, old age, male sex, presence of DM and CKD, and high FIB-4 index were found to be the risk factors for both severe clinical course and mortality (Table 2). The patients were further classified into four groups depending on the presence of DM and the FIB-4 index (Table 3). The patients with a high FIB4 index with DM were the oldest, followed by those with a high FIB-4 index without DM, low FIB-4 index with DM, and low FIB-4 index without DM (Table 3). The rates of mortality and severe clinical course, as well as the duration of hospital stay and quarantine, followed the same trend (Table 3). The patients with DM and patients with a high FIB-4 index exhibited a higher mortality than did those without DM and those with a lower FIB-4 index, respectively, as illustrated by the cumulative mortality (HR, 2.66 and 4.20, respectively) (Fig. 1A, B).

\section{Synergistic contribution of a high FIB-4 index and the} presence of DM as a predictor of COVID-19 mortality

Notably, the patients with both DM and high FIB-4 index ( $n=$ 88 ) had a significantly higher mortality than the other patients (Table 3, Fig. 1C). To determine the independent effect of the presence of DM on mortality, we evaluated mortality according to the presence of DM in the patients with a low FIB-4 index (Fig. 2A) and a high FIB-4 index (Fig. 2B). In the low FIB-4 index group, the presence of DM did not affect the mortality (HR, 0.98; 95\% confidence interval [CI], 0.28 to 3.46 ) (Fig. 2A). However, in the high FIB-4 index group, the presence of DM was associated with a higher mortality (HR, 3.28; 95\% CI, 1.84 to 5.83) (Fig. 2B). In the analysis of mortality according to the FIB-4 index in the patients without DM (Fig. 3A) and those with DM (Fig. 3B), we observed that a high FIB-4 index increased the mortality in the patients with DM (HR, 6.09; $95 \%$ CI, 2.38 to 15.56) compared with that in those without DM (HR, $2.63 ; 95 \% \mathrm{CI}, 1.05$ to 6.60$)$.

\section{FIB-4 index and presence of DM affect the severity and clinical course in patients with COVID-19}

In addition, the odds ratio (OR) for a severe clinical course (Fig. 4A) and the composite of severe clinical course and mortality (Fig. 4B) was the highest in the patients with a high FIB-4 index and DM (OR, 7.61; 95\% CI, 3.95 to 14.65 for severe clinical course; and OR, 11.24; $95 \%$ CI, 5.90 to 21.41 for composite of

Table 2. Multivariate Model for Severe Clinical Course and Death in COVID-19 Patients

\begin{tabular}{|c|c|c|c|c|}
\hline \multirow{2}{*}{ Variable } & \multicolumn{2}{|c|}{ Univariate model } & \multicolumn{2}{|c|}{ Multivariate model } \\
\hline & Severe clinical course & Death & Severe clinical course & Death \\
\hline Age & $1.06(1.05-1.08)^{\mathrm{a}}$ & $1.09(1.07-1.12)^{\mathrm{a}}$ & $1.04(1.02-1.06)^{\mathrm{a}}$ & $1.09(1.06-1.12)^{\mathrm{a}}$ \\
\hline Gender & $2.38(1.66-3.43)^{\mathrm{a}}$ & $3.61(2.19-5.97)^{\mathrm{a}}$ & $1.90(1.25-2.89)^{\mathrm{a}}$ & $3.82(2.08-7.00)^{\mathrm{a}}$ \\
\hline Hypertension & $3.19(2.21-4.62)^{\mathrm{a}}$ & $2.58(1.58-4.20)^{\mathrm{a}}$ & $1.64(1.05-2.58)^{\mathrm{b}}$ & $0.97(0.52-1.80)$ \\
\hline Heart disease & $1.65(0.89-3.05)$ & $1.51(0.67-3.43)$ & $0.64(0.31-1.31)$ & $0.50(0.19-1.30)$ \\
\hline Cerebrovascular disease & $1.42(0.72-2.79)$ & $1.47(0.61-3.53)$ & $0.61(0.28-1.33)$ & $0.61(0.22-1.69)$ \\
\hline Chronic kidney disease & $4.76(2.29-9.88)^{\mathrm{a}}$ & $4.89(2.11-11.32)^{\mathrm{a}}$ & $2.67(1.17-6.12)^{\mathrm{b}}$ & $3.16(1.14-8.75)^{b}$ \\
\hline Chronic lung disease & $1.52(0.81-2.85)$ & $3.12(1.59-6.13)^{\mathrm{a}}$ & $1.32(0.64-2.71)$ & $3.00(1.30-6.94)^{b}$ \\
\hline Cancer & $2.83(1.56-5.14)^{\mathrm{a}}$ & $3.01(1.45-6.23)^{\mathrm{a}}$ & $2.05(1.03-4.11)^{\mathrm{b}}$ & $1.94(0.79-4.79)$ \\
\hline $\mathrm{DM}$ & $3.22(2.20-4.70)^{\mathrm{a}}$ & $5.27(3.21-8.65)^{\mathrm{a}}$ & $1.69(1.08-2.64)^{\mathrm{b}}$ & $3.33(1.83-6.05)^{\mathrm{a}}$ \\
\hline FIB-4 index & $1.55(1.41-1.70)^{\mathrm{a}}$ & $1.43(1.30-1.57)^{\mathrm{a}}$ & $1.35(1.23-1.48)^{\mathrm{a}}$ & $1.29(1.18-1.41)^{\mathrm{a}}$ \\
\hline
\end{tabular}

Data were analyzed using multivariate logistic regression and values are expressed as odds ratio ( $95 \%$ confidence interval). Model was adjusted for age, sex, and underlying diseases. Severe clinical course defined as necessity for the use of a high-flow nasal cannula, mechanical ventilation, continuous renal replacement therapy, or extracorporeal membrane oxygenation or admission to an intensive care unit.

COVID-19, coronavirus disease 2019; DM, diabetes mellitus; FIB-4, fibrosis-4.

${ }^{\mathrm{a}} P<0.01 ;{ }^{\mathrm{b}} P<0.05$. 
Table 3. Baseline Characteristics in Patients Stratified According to Diabetes Mellitus and FIB-4 Index

\begin{tabular}{|c|c|c|c|c|c|}
\hline \multirow{2}{*}{ Characteristic } & \multicolumn{2}{|c|}{ Low FIB-4 index } & \multicolumn{2}{|c|}{ High FIB-4 index } & \multirow{2}{*}{$P$ value } \\
\hline & Non-DM $(n=621)$ & $\operatorname{DM}(n=129)$ & Non-DM $(n=181)$ & $\operatorname{DM}(n=88)$ & \\
\hline Age, yr & $51.2 \pm 16.3$ & $64.6 \pm 10.8$ & $74.2 \pm 10.6$ & $74.8 \pm 8.8$ & $<0.01$ \\
\hline Male sex & $178(28.7)$ & $61(47.3)$ & $79(43.6)$ & $34(38.6)$ & $<0.01$ \\
\hline FIB-4 index & $1.22 \pm 0.63$ & $1.41 \pm 0.65$ & $4.44 \pm 3.45$ & $5.15 \pm 5.11$ & $<0.01$ \\
\hline \multicolumn{6}{|l|}{ Co-morbidity } \\
\hline Hypertension & $138(22.2)$ & $69(53.5)$ & $82(45.3)$ & $67(76.1)$ & $<0.01$ \\
\hline Heart disease & $17(2.7)$ & $16(12.4)$ & $30(16.6)$ & $8(9.1)$ & $<0.01$ \\
\hline Cerebro-vascular disease & $17(2.7)$ & 15 (11.7) & $23(12.8)$ & $7(8.0)$ & $<0.01$ \\
\hline Chronic kidney disease & $6(1.0)$ & $11(8.5)$ & $8(4.4)$ & $7(8.0)$ & $<0.01$ \\
\hline Chronic lung disease & $38(6.1)$ & $6(4.7)$ & $16(8.8)$ & $10(11.4)$ & 0.08 \\
\hline Cancer & $27(4.3)$ & $9(7.0)$ & $15(8.3)$ & $8(9.1)$ & 0.01 \\
\hline
\end{tabular}

Laboratory findings on admission

\begin{tabular}{|c|c|c|c|c|c|}
\hline HbAlc, $\%$ & $5.68 \pm 0.48$ & $7.72 \pm 1.94$ & $5.81 \pm 0.77$ & $7.51 \pm 1.47$ & $<0.01$ \\
\hline Glucose, mg/dL & $107.2 \pm 29.1$ & $186.3 \pm 105.4$ & $116.2 \pm 30.9$ & $182.4 \pm 100.1$ & $<0.01$ \\
\hline $\mathrm{WBC}, 10^{3} / \mu \mathrm{L}$ & $5.25 \pm 1.95$ & $6.97 \pm 3.60$ & $6.02 \pm 3.34$ & $6.79 \pm 3.80$ & $<0.01$ \\
\hline $\mathrm{Hb}, \mathrm{g} / \mathrm{dL}$ & $12.9 \pm 1.54$ & $12.2 \pm 1.66$ & $12.3 \pm 1.72$ & $12.2 \pm 1.79$ & $<0.01$ \\
\hline PLT, $10^{3} / \mu \mathrm{L}$ & $243.4 \pm 81.4$ & $276.0 \pm 96.5$ & $167.2 \pm 58.0$ & $168.8 \pm 70.4$ & 0.35 \\
\hline $\mathrm{CRP}, \mathrm{mg} / \mathrm{dL}$ & $4.9 \pm 18.0$ & $10.2 \pm 22.7$ & $12.8 \pm 26.6$ & $12.0 \pm 13.5$ & $<0.01$ \\
\hline Total protein, g/dL & $6.84 \pm 0.51$ & $6.78 \pm 0.65$ & $6.54 \pm 0.61$ & $6.56 \pm 0.76$ & $<0.01$ \\
\hline Albumin, g/dL & $4.05 \pm 0.42$ & $3.78 \pm 0.51$ & $3.57 \pm 0.46$ & $3.46 \pm 0.57$ & $<0.01$ \\
\hline $\mathrm{eGFR}, \mathrm{mL} / \mathrm{min} / 1.73 \mathrm{~m}^{2}$ & $103.3 \pm 31.9$ & $84.1 \pm 34.2$ & $81.8 \pm 35.1$ & $71.5 \pm 31.6$ & $<0.01$ \\
\hline AST, IU/L & $24.3 \pm 10.3$ & $25.7 \pm 12.7$ & $40.3 \pm 23.0$ & $43.7 \pm 28.6$ & $<0.01$ \\
\hline ALT, IU/L & $22.9 \pm 13.3$ & $23.2 \pm 12.9$ & $23.4 \pm 15.0$ & $23.4 \pm 14.7$ & 0.96 \\
\hline LDH, IU/L & $427.5 \pm 158.8$ & $446.9 \pm 223.7$ & $501.4 \pm 222.9$ & $656.3 \pm 577.3$ & $<0.01$ \\
\hline \multicolumn{6}{|l|}{ Treatment } \\
\hline ICU & $22(3.5)$ & $16(12.4)$ & $40(22.1)$ & $34(38.6)$ & $<0.01$ \\
\hline High flow $\mathrm{O}_{2}$ & $11(1.8)$ & $8(6.2)$ & $34(18.8)$ & $22(25.0)$ & $<0.01$ \\
\hline Ventilator & $9(1.4)$ & $9(7.0)$ & $22(12.2)$ & $22(25.0)$ & $<0.01$ \\
\hline CRRT & $3(0.5)$ & $3(2.3)$ & $2(1.1)$ & $10(11.4)$ & $<0.01$ \\
\hline ECMO & $2(0.3)$ & $2(1.6)$ & $2(1.1)$ & $6(6.8)$ & $<0.01$ \\
\hline \multicolumn{6}{|l|}{ Duration } \\
\hline Confirmation-release ${ }^{\mathrm{a}}$, day & $28.0 \pm 13.2$ & $31.5 \pm 13.0$ & $34.8 \pm 13.4$ & $34.8 \pm 16.1$ & $<0.01$ \\
\hline Hospital length ${ }^{\mathrm{a}}$, day & $23.0 \pm 12.7$ & $26.8 \pm 12.5$ & $30.9 \pm 13.4$ & $33.0 \pm 17.9$ & $<0.01$ \\
\hline \multicolumn{6}{|l|}{ Outcome } \\
\hline Release & $584(94.0)$ & $118(91.5)$ & $142(78.5)$ & $52(59.1)$ & $<0.01$ \\
\hline Severe clinical course & $28(4.5)$ & $18(14.0)$ & $52(28.7)$ & $39(44.3)$ & $<0.01$ \\
\hline Death & $8(1.3)$ & $6(4.7)$ & $24(13.3)$ & $33(37.5)$ & $<0.01$ \\
\hline
\end{tabular}

Values are expressed as mean \pm standard deviation or number $(\%)$. All $P$ values were calculated using the analysis of variance or the chi-square test. FIB-4, fibrosis-4; DM, diabetes mellitus; HbA1c, hemoglobin A1c; WBC, white blood cell; Hb, hemoglobin; PLT, platelet; CRP, C-reactive protein; eGFR, estimated glomerular filtration rate; AST, aspartate aminotransferase; ALT, alanine aminotransferase; LDH, lactate dehydrogenase; ICU, intensive care unit; CRRT, continuous renal replacement therapy; ECMO, extracorporeal membrane oxygenation.

${ }^{a}$ Only includes patients who had been released from quarantine. 

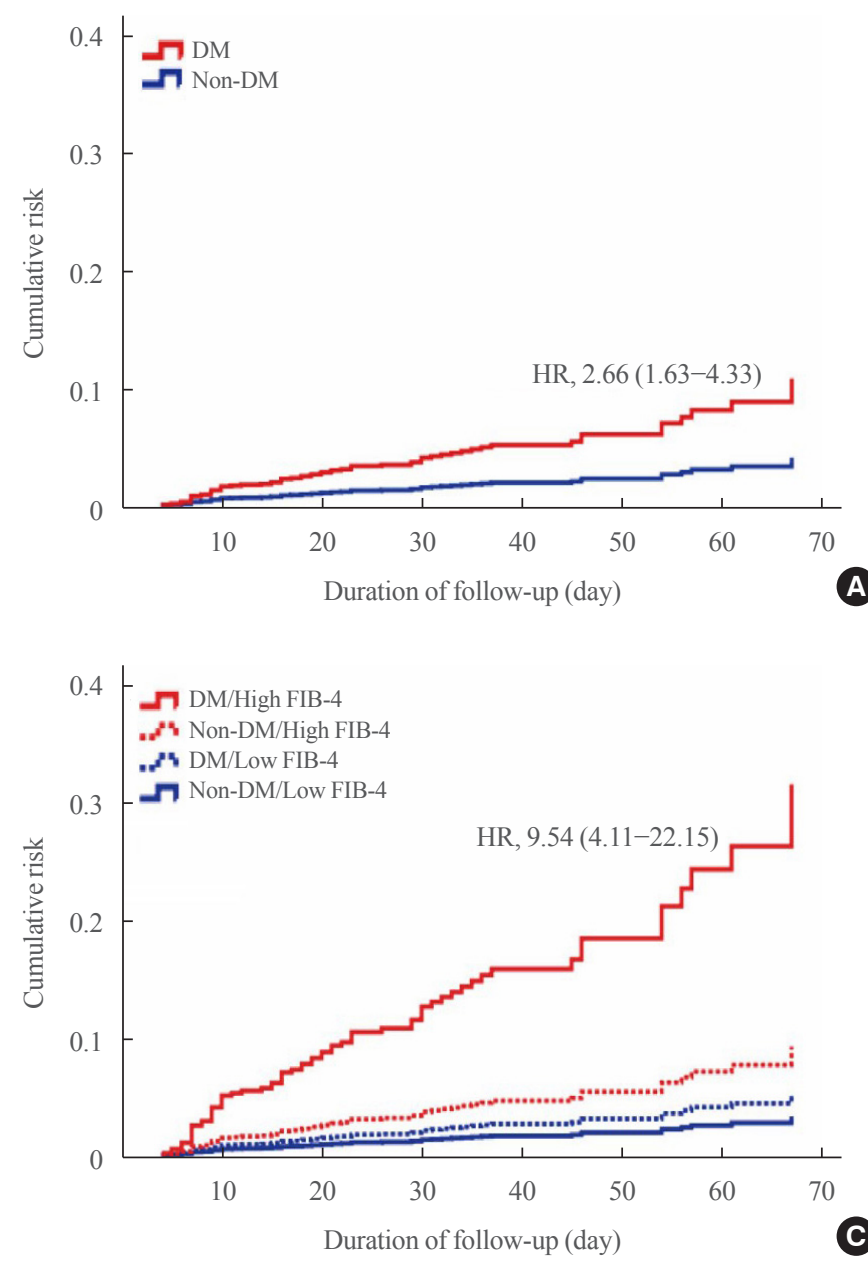

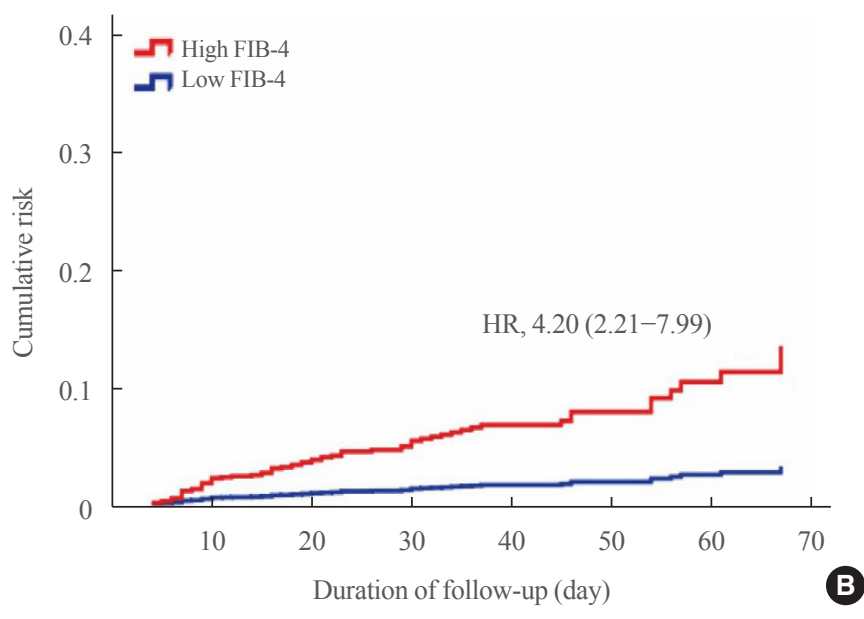

Fig. 1. Mortality of coronavirus disease 2019 (COVID-19) patients according to (A) diabetes mellitus (DM), (B) fibrosis-4 (FIB-4) index, and (C) diabetes mellitus (DM) and FIB-4 index. (A) Red line, patients with diabetes; blue line, patients without diabetes. (B) Red line, patients with high FIB-4 index; blue line, patients with low FIB-4 index. (C) Red solid line, patents with diabetes and high FIB4 index; red dashed line, non-diabetes patients with high FIB-4 index; blue solid line, patents with diabetes and low FIB-4 index; blue dashed line, non-diabetes patients with low FIB-4 index. Data were analyzed using Cox proportional hazards model and all data are expressed as hazard ratio (HR) (95\% confidence interval). Model was adjusted for age, sex, and underlying diseases.

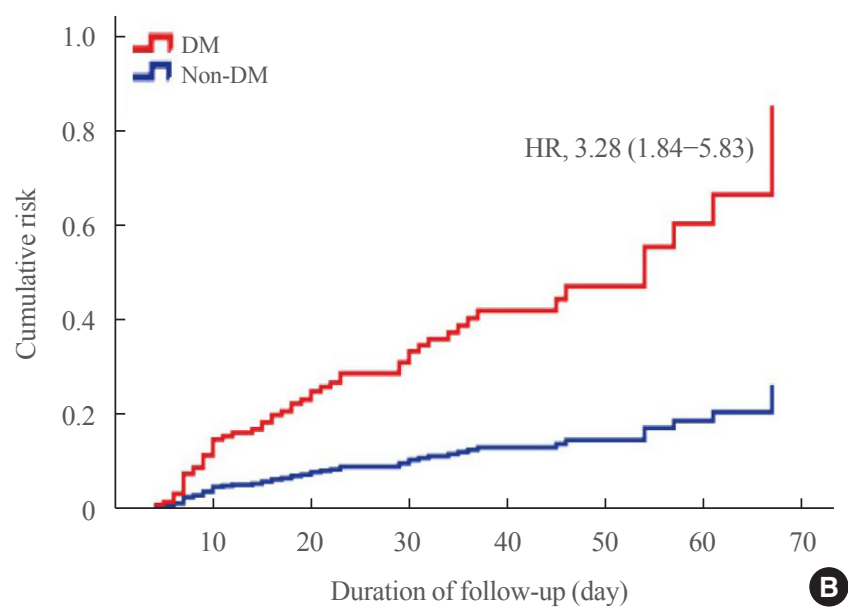

Fig. 2. Mortality of coronavirus disease 2019 (COVID-19) according to the presence of diabetes mellitus (DM) in patient (A) with low fibrosis-4 (FIB-4) index and (B) with high FIB-4 index. Red line, patients with diabetes; blue line, patients without diabetes. Data were analyzed using Cox proportional hazards model and all data are expressed as hazard ratio (HR) (95\% confidence interval). Model was adjusted for age, sex, and underlying diseases. 

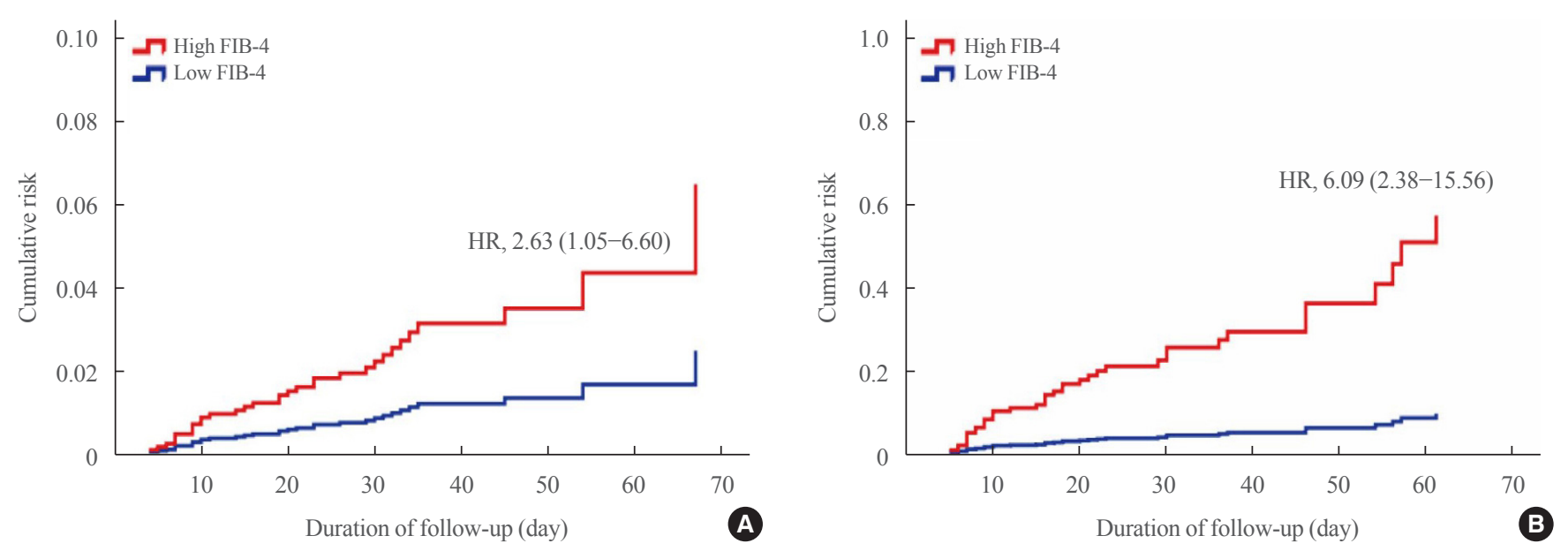

Fig. 3. Mortality of coronavirus disease 2019 (COVID-19) according to fibrosis-4 (FIB-4) index in patient (A) without diabetes mellitus (DM) and (B) with DM. Red line, patients with high FIB-4 index; blue line, patients with low FIB-4 index. Data were analyzed using Cox proportional hazards model all data are expressed as hazard ratio (HR) (95\% confidence interval). Model was adjusted for age, sex, and underlying diseases.
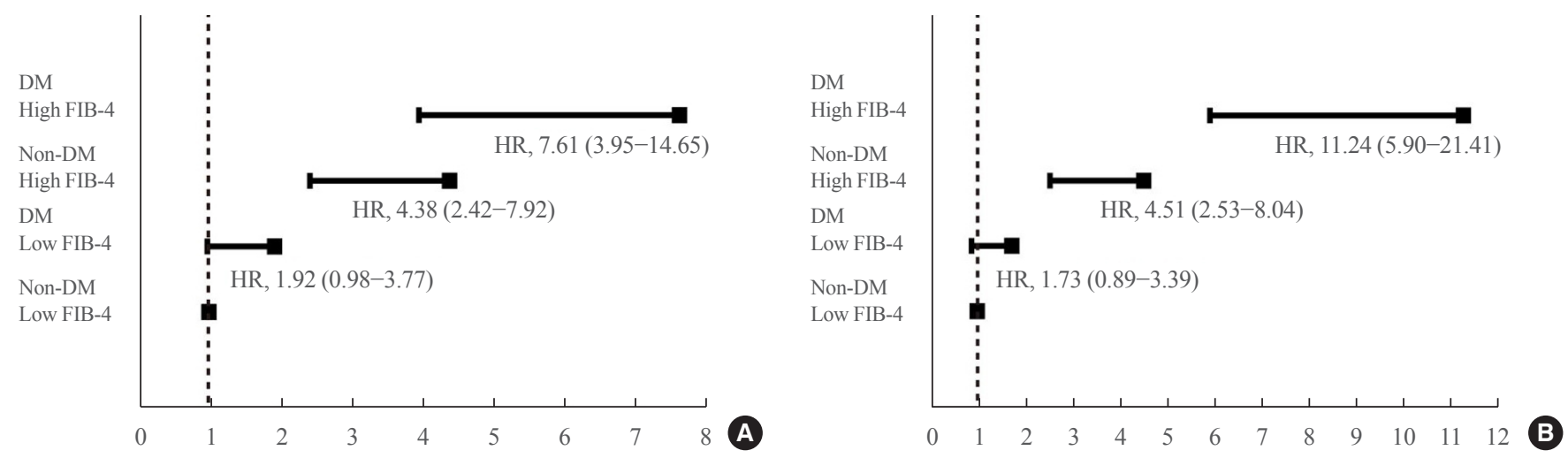

Fig. 4. Odds ratio (OR) of severe clinical course (A) and severe clinical course or death (B) according to patients group stratified according to the presence of diabetes mellitus (DM) or fibrosis-4 (FIB-4) index. Data were analyzed using multivariate logistic regression and all data are expressed as OR (95\% confidence intervals). Model was adjusted for age, sex, and underlying diseases. HR, hazard ratio.

severe clinical course and mortality). The duration of quarantine, defined as the period from confirmation of COVID-19 to release from quarantine, and the duration of hospital stay were compared among the four groups. The duration of quarantine was the longest in the high FIB-4 index with DM group, although no significant difference was observed (32.5 \pm 1.9 days) (Fig. 5A). Meanwhile, the duration of hospital stay was significantly longer in the high FIB-4 index groups (28.5 \pm 1.2 and $30.8 \pm 1.9$ days for those without DM and with DM, respectively) than in the low FIB-4 index group without DM $(23.8 \pm 0.6$ days), even after adjusting for multiple confounding factors (Fig. 5B). Collectively, the presence of DM and a high baseline FIB-4 index were related with worse COVID-19 clinical outcome in a complementary and independent manner.

\section{DISCUSSION}

Herein, we showed that both presence of DM and high FIB-4 index are related with higher mortality of COVID-19 infection. This study was an extension of our previous report, which showed that patients with DM have a higher mortality [9]. In addition to these results, we identified that the patients with DM had a higher FIB-4 index, and among those with a low FIB-4 index, DM exhibited a minimal relationship with mortality. In contrast, regardless of the presence of DM, a high FIB-4 index reflected a higher mortality. In particular, the patients with DM with a high FIB-4 index tended to show the most severe clinical course and mortality than did those with DM only or a high FIB-4 index only, suggesting a complementary role in predict- 

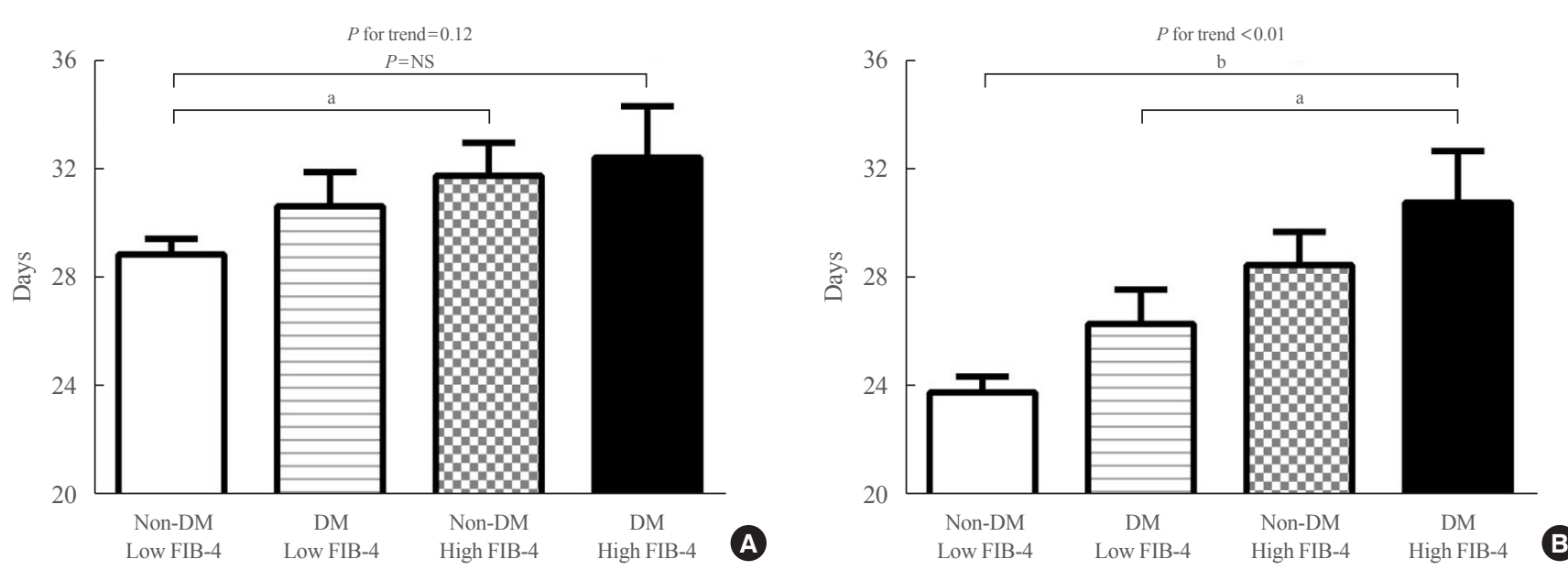

Fig. 5. The estimated mean of duration of confirmation to (A) release and (B) hospital length. Data were analyzed using general linear model and all data are expressed as the mean \pm standard error. Model was adjusted for age, sex, and underlying diseases. NS, not significant; DM, diabetes mellitus; FIB-4, fibrosis-4. ${ }^{\mathrm{a}} P<0.01$; ${ }^{\mathrm{b}} P<0.05$.

ing COVID-19 mortality.

Several pieces of evidence suggest the prognostic value of the FIB-4 index in COVID-19 cases [12,16,24,25]. Liver injury in COVID-19 cases has been observed more frequently in severe cases [26]. Although the mechanism remains elusive, it is very likely that liver enzyme level elevation may be related to aberrant cytokine release, which is frequently observed in severe pneumonia [27]. Angiotensin-converting enzyme 2 (ACE-2) receptors have been reported to be present in both cholangiocytes and hepatocytes. Therefore, it is likely that this leads to cellular injury [28]. Conversely, advanced liver disease accompanies persistent stimulation of immune cells by pathogen-associated molecular patterns or damage-associated molecular patterns that are responsible for the activation of immune cells [29]. Immune cell-driven upregulation of cytokine production activates additional inflammatory cells, forming a low-grade inflammatory milieu [29].

FIB-4 index is a non-invasive panel to assess underlying liver fibrosis, and recently widely accepted as inexpensive and accurate tool in patients with hepatic disorders such as viral hepatitis and NAFLD/NASH. Especially, NAFLD/NASH encompass various stages from simple steatosis to cirrhosis, and progression to advanced stage such as steatohepatitis and fibrosis is known to increase morbidity and mortality as well as cardiovascular risk. Recent guideline recommended scoring for advanced fibrosis in NAFLD suspected individuals [20,30], and FIB-4 is one of the constitute predictive models. Interestingly, FIB-4 was closely associated with the disease severity and mortality of extrahepatic disorders as well as liver stiffness in previous studies
[13-15]. As we commented above, worse outcomes have been reported in patients with underlying liver disease and cirrhosis, it is easily speculated the subjects having higher FIB-4 are vulnerable to SARS-CoV-2 infection. Consistently to our results, Park et al. [16] demonstrated that the survival rate was significantly lower in high FIB-4 group compare to the low FIB-4 group. These findings supported the predictive role of FIB-4 as we shown in our study, and would be useful to effective delivery of healthcare resources in COVID-19 pandemic era.

Our results show that although DM alone is a critical risk factor, the addition of the FIB-4 index can more specifically predict COVID-19 mortality and severity. This trend was valid even after adjusting for age, sex, and underlying disease. This is consistent with recent findings from previous studies in which the proportion of severe COVID-19 cases was higher in patients with MAFLD [31]. The risk was reported to have a wide range of OR (1.10 to 31.2$)$, and this variability among studies is probably attributed to the different study designs, prevalence of COVID-19, quarantine strategies, and definitions of MAFLD or NAFLD/NASH. Nevertheless, the findings from all relevant studies indicate that hepatic fibrosis is a crucial factor in determining the outcomes of patients with SARS-CoV-2 infection, and the risk is synergistically increased in the presence of DM. In this regard, it can be postulated that among patients with DM, those with a high FIB-4 index are at a higher risk of developing a more severe inflammatory status.

Because of the considerable increase in the number of patients with COVID-19 in Daegu, we had no opportunity to examine the prevalence of NAFLD among them either via medical history- 
taking or via abdominal ultrasonography. Therefore, we were unable to discriminate NAFLD from infection-related acute liver injury. Although we excluded patients who had ALT levels of $\geq$ $80 \mathrm{IU} / \mathrm{L}$ at the time of admission or died within 3 days from diagnosis with COVID-19 who were more likely to have COVID19-related liver injury, these arbitrary criteria cannot fully exclude acute liver injury. Nevertheless, it has been reported that even in populations that are clinically not diagnosed with NAFLD, the FIB-4 index correlates with disease severity [32,33], suggesting that apart from the presence of NAFLD or NASH, the FIB-4 index per se might serve as a disease severity marker.

Our study had some limitations. First, because of a shortage of infrastructure and medical staff, there was incomplete documentation regarding exposure history and lack of timely laboratory examinations being conducted. In particular, as mentioned above, the acquisition of demographic information to account for NAFLD/NASH as a comorbidity was not performed. Second, of the confirmed cases of COVID-19 in Daegu, 65.6\% were associated with a single religious group, and these patients were admitted to university hospitals because of their disease severity; therefore, the data are more likely to be representative of patients with COVID-19 with moderate-to-severe disease, which indicates a lack of heterogeneity. Lastly, other prognostic markers, such as the D-dimer, lactate dehydrogenase, or ferritin level, were not measured and compared with the FIB-4 index.

Nevertheless, our study findings strongly suggest that DM and the FIB-4 index can play independent roles in the prediction of COVID-19 mortality and disease severity. Patients with DM and a high FIB-4 index could have an almost 10-fold higher mortality risk than those without DM and a low FIB-4 index. Furthermore, it is noteworthy that among patients with DM, the FIB-4 index could be used in further stratifying the risk. Therefore, special attention is required for patients with DM and a high FIB-4 index upon admission.

\section{CONFLICTS OF INTEREST}

No potential conflict of interest relevant to this article was reported.

\section{ACKNOWLEDGMENTS}

We would like to express sincere gratitude to all the members of the Daegu-Gyeongbuk Diabetes and Endocrinology Society who actively participated in the study.

\section{AUTHOR CONTRIBUTIONS}

Conception or design: S.W.K., J.H.J., J.S.M., M.K.K. Acquisition, analysis, or interpretation of data: S.W.K., J.H.J., J.S.M., M.K.K. Drafting the work or revising: S.W.K., J.H.J., J.S.M., M.K.K. Final approval of the manuscript: S.W.K., J.H.J., J.S.M., M.K.K.

\section{ORCID}

Sung-Woo Kim https://orcid.org/0000-0001-5354-8430

Jae-Han Jeon https://orcid.org/0000-0002-9217-968X

Jun Sung Moon https://orcid.org/0000-0003-1569-3068

Mi Kyung Kim https://orcid.org/0000-0001-5750-3598

\section{REFERENCES}

1. Fu L, Wang B, Yuan T, Chen X, Ao Y, Fitzpatrick T, et al. Clinical characteristics of coronavirus disease 2019 (COVID-19) in China: a systematic review and meta-analysis. J Infect 2020;80:656-65.

2. Kim DW, Byeon KH, Kim J, Cho KD, Lee N. The correlation of comorbidities on the mortality in patients with covid-19: an observational study based on the Korean National Health Insurance Big Data. J Korean Med Sci 2020;35:e243.

3. Kumar A, Arora A, Sharma P, Anikhindi SA, Bansal N, Singla $\mathrm{V}$, et al. Is diabetes mellitus associated with mortality and severity of COVID-19? A meta-analysis. Diabetes Metab Syndr 2020;14:535-45.

4. Cai Q, Huang D, Yu H, Zhu Z, Xia Z, Su Y, et al. COVID-19: abnormal liver function tests. J Hepatol 2020;73:566-74.

5. Guan WJ, Ni ZY, Hu Y, Liang WH, Ou CQ, He JX, et al. Clinical characteristics of coronavirus disease 2019 in China. N Engl J Med 2020;382:1708-20.

6. Younossi Z, Anstee QM, Marietti M, Hardy T, Henry L, Eslam M, et al. Global burden of NAFLD and NASH: trends, predictions, risk factors and prevention. Nat Rev Gastroenterol Hepatol 2018;15:11-20.

7. Eslam M, Newsome PN, Sarin SK, Anstee QM, Targher G, Romero-Gomez M, et al. A new definition for metabolic dysfunction-associated fatty liver disease: an international expert consensus statement. J Hepatol 2020;73:202-9.

8. Fouad Y, Waked I, Bollipo S, Gomaa A, Ajlouni Y, Attia D. What's in a name? Renaming 'NAFLD' to 'MAFLD'. Liver Int 2020;40:1254-61.

9. Kim MK, Jeon JH, Kim SW, Moon JS, Cho NH, Han E, et 
al. The clinical characteristics and outcomes of patients with moderate-to-severe coronavirus disease 2019 infection and diabetes in Daegu, South Korea. Diabetes Metab J 2020;44: 602-13.

10. Zhou YJ, Zheng KI, Wang XB, Yan HD, Sun QF, Pan KH, et al. Younger patients with MAFLD are at increased risk of severe COVID-19 illness: a multicenter preliminary analysis. J Hepatol 2020;73:719-21.

11. Gao F, Zheng KI, Wang XB, Yan HD, Sun QF, Pan KH, et al. Metabolic associated fatty liver disease increases coronavirus disease 2019 disease severity in nondiabetic patients. J Gastroenterol Hepatol 2021;36:204-7.

12. Targher G, Mantovani A, Byrne CD, Wang XB, Yan HD, Sun QF, et al. Risk of severe illness from COVID-19 in patients with metabolic dysfunction-associated fatty liver disease and increased fibrosis scores. Gut 2020;69:1545-7.

13. Saito Y, Okumura Y, Nagashima K, Fukamachi D, Yokoyama K, Matsumoto N, et al. Impact of the fibrosis-4 index on risk stratification of cardiovascular events and mortality in patients with atrial fibrillation: findings from a Japanese multicenter registry. J Clin Med 2020;9:584.

14. Jin JL, Zhang HW, Cao YX, Liu HH, Hua Q, Li YF, et al. Liver fibrosis scores and coronary atherosclerosis: novel findings in patients with stable coronary artery disease. Hepatol Int 2021;15:413-23.

15. Schonmann Y, Yeshua H, Bentov I, Zelber-Sagi S. Liver fibrosis marker is an independent predictor of cardiovascular morbidity and mortality in the general population. Dig Liver Dis 2021;53:79-85.

16. Park JG, Kang MK, Lee YR, Song JE, Kim NY, Kweon YO, et al. Fibrosis-4 index as a predictor for mortality in hospitalised patients with COVID-19: a retrospective multicentre cohort study. BMJ Open 2020;10:e041989.

17. Lopez-Mendez I, Aquino-Matus J, Gall SM, Prieto-Nava JD, Juarez-Hernandez E, Uribe M, et al. Association of liver steatosis and fibrosis with clinical outcomes in patients with SARS-CoV-2 infection (COVID-19). Ann Hepatol 2021;20: 100271.

18. Forlano R, Mullish BH, Mukherjee SK, Nathwani R, Harlow $\mathrm{C}$, Crook $\mathrm{P}$, et al. In-hospital mortality is associated with inflammatory response in NAFLD patients admitted for COVID-19. PLoS One 2020;15:e0240400.

19. Kim MK, Ko SH, Kim BY, Kang ES, Noh J, Kim SK, et al. 2019 Clinical practice guidelines for type 2 diabetes mellitus in Korea. Diabetes Metab J 2019;43:398-406.

20. Vilar-Gomez E, Chalasani N. Non-invasive assessment of non-alcoholic fatty liver disease: clinical prediction rules and blood-based biomarkers. J Hepatol 2018;68:305-15.

21. Chung SM, Lee YY, Ha E, Yoon JS, Won KC, Lee HW, et al. The risk of diabetes on clinical outcomes in patients with coronavirus disease 2019: a retrospective cohort study. Diabetes Metab J 2020;44:405-13.

22. Guo W, Li M, Dong Y, Zhou H, Zhang Z, Tian C, et al. Diabetes is a risk factor for the progression and prognosis of COVID-19. Diabetes Metab Res Rev 2020;36:e3319.

23. Cristelo C, Azevedo C, Marques JM, Nunes R, Sarmento B. SARS-CoV-2 and diabetes: new challenges for the disease. Diabetes Res Clin Pract 2020;164:108228.

24. Li Y, Regan J, Fajnzylber J, Coxen K, Corry H, Wong C, et al. Liver fibrosis index fib-4 is associated with mortality in COVID-19. Hepatol Commun 2020;5:434-45.

25. Sterling RK, Oakes T, Gal TS, Stevens MP, DeWit M, Sanyal AJ. The fibrosis-4 index is associated with need for mechanical ventilation and 30-day mortality in patients admitted with coronavirus disease 2019. J Infect Dis 2020;222:1794-7.

26. Huang C, Wang Y, Li X, Ren L, Zhao J, Hu Y, et al. Clinical features of patients infected with 2019 novel coronavirus in Wuhan, China. Lancet 2020;395:497-506.

27. Fan Z, Chen L, Li J, Cheng X, Yang J, Tian C, et al. Clinical features of COVID-19-related liver functional abnormality. Clin Gastroenterol Hepatol 2020;18:1561-6.

28. Xu L, Liu J, Lu M, Yang D, Zheng X. Liver injury during highly pathogenic human coronavirus infections. Liver Int 2020;40:998-1004.

29. Li D, Ding X, Xie M, Tian D, Xia L. COVID-19-associated liver injury: from bedside to bench. J Gastroenterol 2021;56: 218-30.

30. Chalasani N, Younossi Z, Lavine JE, Charlton M, Cusi K, Rinella M, et al. The diagnosis and management of nonalcoholic fatty liver disease: practice guidance from the American Association for the Study of Liver Diseases. Hepatology 2018;67:328-57.

31. Sy-Janairo ML, Y Cua IH. Association of metabolic-associated fatty liver disease and risk of severe coronavirus disease 2019 illness. JGH Open 2020;5:4-10.

32. Parikh NS, Kamel H, Navi BB, Iadecola C, Merkler AE, Jesudian A, et al. Liver fibrosis indices and outcomes after primary intracerebral hemorrhage. Stroke 2020;51:830-7.

33. Cao YX, Zhang M, Zhang HW, Jin JL, Liu HH, Zhang Y, et al. Impact of liver fibrosis score on prognosis in patients with previous myocardial infarction: a prospective cohort study. Liver Int 2021;41:1294-304. 